\title{
Estamos prontos para utilizar a avaliação em pares na Educação a Distância? Um estudo de caso em um curso de especialização
}

\author{
Marcelo Werneck Barbosa ${ }^{1}$, Maria Augusta Vieira Nelson ${ }^{1}$ \\ ${ }^{1}$ Instituto de Ciências Exatas e Informática - Pontifícia Universidade Católica de Minas \\ Gerais (PUC Minas) \\ $\{$ mwerneck, guta\} @pucminas.br
}

\begin{abstract}
Resumo. Este trabalho apresentou o estudo de um caso de aplicação da técnica de avaliação em pares como mecanismo de avaliação em um curso de especialização à distância. O estudo foi realizado por meio do preenchimento de um questionário pelos alunos e também pela análise de dados do próprio curso estudado. A maioria dos alunos considerou a avaliação realizada pelos colegas como justa ou muito justa e que ela contribui com seu aprendizado. Entretanto, este trabalho identificou indícios que uma parte considerável dos alunos não se dedicou completamente à avaliação. $O$ trabalho ainda apresenta lições aprendidas da execução deste processo.
\end{abstract}

Palavras-chave: educação à distância, avaliação em pares, mecanismos de avaliação.

\section{Are we ready to use peer reviews in Distance Education? A case study in a post-graduation course}

\begin{abstract}
This work presents a case study of the adoption of peer review as an assessment technique in a virtual post-graduation course. The study was performed by analyzing data of a questionnaire filled in by students and data on the course execution itself. Most students considered the peer review as fair or very fair and that it positively contributed to their learning. However, this study identified evidence that a considerable part of the students did not dedicate themselves fully to the assessment activity. This work also presents lessons learned on this whole process.
\end{abstract}

Keywords: distance education, peer review, assessment mechanisms.

\section{Introdução}

A avaliação das aprendizagens pode ser entendida como um processo eminentemente social e formativo orientado para os objetivos pedagógicos e competências a atingir pelos alunos. No entanto, esta deverá ser suficientemente flexível e diversificada para ir ao encontro das necessidades e características do público-alvo de acordo com o ambiente em que o processo de ensino e de aprendizagem ocorre (Balula, 2005). 
Todo processo de aprendizado deve envolver alguma forma de avaliação do conhecimento, competências e/ou habilidades alcançadas. É também notório o fato de que avaliar o trabalho de outra pessoa pode ser uma técnica poderosa de aprendizado (Zenha-Rela e Carvalho, 2006). No ensino presencial, há uma clara distinção entre os papéis do professor e alunos de tal forma que a avaliação do aprendizado é uma tarefa indiscutivelmente do professor. Em um contexto de Educação a Distância (EaD), é comum que se transponham estratégias do ensino presencial sem se ter em conta as características do novo ambiente, ou seja, negligenciando o fato de o sucesso da sua aplicação depender, em grande parte, da adequação que se faz ao meio e às características dos próprios participantes (Balula, 2013). Uma das possíveis formas de se implementar avaliações em cursos a distância é por meio de avaliações por pares.

A avaliação por pares, ou revisão por pares, é um método estático de verificação no qual o produto de um trabalho é examinado por uma pessoa que não seja o autor do mesmo, com o propósito de detectar defeitos. Em uma revisão por pares são usados critérios objetivos para a avaliação (Softex, 2016). A avaliação em pares pode trazer um sentimento de se pertencer a um grupo e é considerada uma boa ideia em termos de aprendizado e retenção dos alunos e interação entre os mesmos (Chetwynd, Gardner e Jefferis, 2013). Pesquisadores destacam o fato de que revisão por pares oferece aos alunos a chance de desenvolver um grupo de capacidades importantes para o desenvolvimento da linguagem e habilidades de escrita e uma maior exposição a ideias (Papadopoulos et al., 2012).

Neste contexto, o objetivo deste trabalho é analisar a utilização de avaliação por pares em Educação a Distância por meio de um estudo de caso em um curso de especialização virtual de uma grande universidade brasileira.

O restante deste artigo está organizado da seguinte forma. A Seção 2 apresenta o referencial teórico, sobretudo os conceitos relacionados a revisão por pares em EaD. A Seção 3 descreve a metodologia utilizada neste estudo enquanto a Seção 4 apresenta os resultados deste trabalho. A Seção 5 apresenta conclusões do trabalho, suas limitações e possibilidades de trabalhos futuros.

\section{Referencial Teórico}

\subsection{A Avaliação em Pares na Educação a Distância}

No ambiente da $\mathrm{EaD}$, é fundamental que as atividades e tarefas propostas aos alunos se constituam de elementos de avaliação com critérios, indicadores e pesos definidos a priori. Dentre os instrumentos mais comumente utilizados neste contexto, podem ser citados: os testes (com questões de resposta fechada e/ou aberta), os trabalhos escritos (individuais ou em grupo), a participação on-line e a auto e heteroavaliação (Balula, 2013). Heteroavaliação é a avaliação dos estudantes sobre a atuação docente (Nascimento, Ferreira e Cordeiro, 2011). Uma forma ainda pouco explorada de avaliação em cursos EaD é a avaliação por pares.

A avaliação por pares é um método instrucional com o objetivo de auxiliar os alunos a construir conhecimento em um domínio específico, enquanto simultaneamente desenvolvem capacidades de revisão (Papadopoulos et al., 2012). As vantagens de 
atividades de avaliação por pares têm sido relatadas em vários estudos. Este tipo de avaliação ajuda os alunos a desenvolver habilidades para, de maneira objetiva e crítica, ler os seus próprios trabalhos. Além de proporcionar aos autores consciência dos pontos fortes e fracos de seus próprios trabalhos, as avaliações por pares podem fornecer comentários bastante específicos e úteis que podem estimular melhores revisões ou melhorias na produção destes alunos (Yoshikawa, Terrano e Yoshikawa, 2012). O uso da avaliação por partes teve forte contribuição dos MOOCs (massive open online cursos - cursos online abertos e massivos). A avaliação por pares apresenta características que a tornam útil para se avaliar perguntas abertas ou trabalhos mais complexos (GarciaAlmirall et al., 2015).

Song e Siriluck (2009) compararam, em um ambiente de avaliação por pares, os comentários fornecidos pelos pares em um ambiente eletrônico com os comentários fornecidos em avaliações feitas de maneira presencial, cara a cara. Os autores observaram que os comentários realizados por meio eletrônico sofrem menos influência da autoridade do professor, desenvolvem a capacidade de aprendizado autônomo no aluno em um ambiente livre de ameaças e aumentam as oportunidades para realização de interações entre os alunos. Em seu estudo, os autores observaram que o grupo que participou das revisões cara a cara produziu mais comentários que o grupo que realizou o processo em meio eletrônico. Entretanto, este último grupo produziu mais comentários orientados à própria revisão. Assim, os alunos que participaram do grupo em um meio eletrônico produziram melhores trabalhos que aqueles do grupo presencial.

Uma das principais considerações mencionadas quando se considera a avaliação por pares em EaD é se a introdução da avaliação pelos pares em um curso reduz o tempo que o professor dedica ao curso. Estudos anteriores mostraram ligeira economia de tempo quando comparada a revisão por pares com um acompanhamento em relação à avaliação tradicional (Gielen et al., 2011). Tais estudos sugerem que o tempo economizado pode ser utilizado em outras tarefas do professor ou tutor. Além disso, a avaliação por pares também é mais facilmente corrigida, pois seus formulários podem ser facilmente interpretados por um computador (Chetwynd, Gardner um e Jefferis, 2013).

Por outro lado, uma das desvantagens de se utilizar a avaliação por pares é a dificuldade de se garantir que todos os alunos tenham a capacidade crítica de julgar de forma eficaz e fazer comentários que contribuem para o aprendizado dos colegas. Níveis de habilidade assimétricos entre os pares podem resultar em desconforto ou atitudes defensivas por parte de alguns alunos ou ainda comportamentos não totalmente sinceros em função de admiração ou amizade com os colegas (Carlson, Berry e Voltmer, 2005).

Foram encontrados na literatura relatos do uso de avaliação por pares em curso $\mathrm{EaD}$, porém, estes são usualmente ligados a atividades de avaliação de escrita dos alunos (Yoshizawa, Terano e Yoshikawa, 2012). Usos mais amplos desta técnica não foram observados e motivam a realização deste trabalho.

\section{Procedimentos Metodológicos}

A metodologia adotada neste trabalho é a de um estudo de caso. Yin (1984) define o estudo de caso como uma pesquisa empírica que investiga um fenômeno contemporâneo em seu contexto natural, em situações em que as fronteiras entre o contexto e o 
fenômeno não são claramente evidentes, utilizando múltiplas fontes de evidência. $\mathrm{O}$ cenário de estudo são as disciplinas de Modelagem de Aplicações Web e Construção de Aplicações Web do curso de especialização em Desenvolvimento de Aplicações Web de uma grande universidade privada brasileira. O curso está disponibilizado em um Ambiente Virtual de Aprendizagem (AVA) no sistema Moodle. O Moodle é utilizado em 193 países, com 400.000 usuários registrados (Muhsen et al., 2014).

O curso de especialização em Desenvolvimento de Aplicações Web busca capacitar os alunos para o domínio das tecnologias Web, em especial aquelas relacionadas ao desenvolvimento de aplicações, bem como para a adoção de melhores práticas em Engenharia de Software para a Web. Além disso, tem-se observado uma busca por novos serviços e aplicações para a Web, que exigem que os profissionais da área, além de serem capazes de lidar com as tecnologias, saibam empregar os métodos, as técnicas e os padrões de projeto da Engenharia de Software para aumentar o grau de sucesso dos seus projetos.

O curso tem uma duração de 3 semestres. Em seu segundo semestre, são oferecidas disciplinas da Engenharia de Software chamadas de Modelagem de Aplicações Web, Projeto de Aplicações Web e, por fim, Construção e implantação de aplicações Web. A disciplina de Modelagem de Aplicações Web trata de temas relacionados à introdução à engenharia de software, como processos e ciclos de vida, assim como requisitos e análise de software. A disciplina de Projeto de Aplicações Web aborda o projeto de sistemas, a arquitetura de software, a arquitetura da informação e projeto de interfaces e usabilidade. A última disciplina do eixo, Construção e Implantação de Aplicações Web cuida de temas da gerência de configuração, testes de software e métricas do desenvolvimento de software.

As disciplinas estão divididas em quatro unidades e têm duração de quatro semanas. Para cada unidade, foram gravadas vídeo-aulas. Os alunos apresentaram suas dúvidas e discussões por meio de fóruns. Como mecanismos de avaliação, cada unidade, ao final, possuiu uma atividade objetiva com seis questões e, ao longo da disciplina, os alunos realizaram a atividade aberta, que foi entregue ao final da disciplina. Esta atividade aberta é o foco deste estudo por ser o trabalho submetido à avaliação por pares. Ao final do semestre, os alunos foram submetidos a uma prova presencial. $\mathrm{O}$ estudo de caso tem o objetivo de introduzir e verificar os resultados da avaliação em pares nas disciplinas.

Como ponto de partida para se introduzir avaliação por pares em um curso, Gielen et al. (2011) sugerem que é importante decidir sobre os objetivos da revisão por pares e, com base nestes objetivos, selecionar os critérios apropriados de qualidade. Tais critérios de qualidade são usualmente elaborados e disponibilizados por meio de uma rubrica. A principal vantagem para os alunos é que diante de uma rubrica eles conhecem desde o primeiro dia do curso as características e exigências que se esperam deles, assim como os critérios de avaliação que serão aplicados (Garcia-Almirall et al., 2015).

A rubrica nas disciplinas foi disponibilizada por meio de um checklist de verificação, com itens de verificação sobre as entregas dos alunos. Os itens foram elaborados considerando características importantes das entregas segundo o professor e literatura da área de Engenharia de Software. A avaliação em pares foi toda realizada no 
AVA. O checklist de verificação foi cadastrado como um formulário no ambiente e estava disponível logo após o encerramento do prazo de envio da atividade. Foram adotadas algumas regras para realização da avaliação por pares:

- À atividade de cada aluno foram alocados pelo AVA 5 revisores aleatórios;

- Havia um prazo máximo para realização da avaliação;

- A avaliação dos trabalhos dos colegas teve um peso de $20 \%$ da nota enquanto a realização da atividade aberta em si teve um peso de $80 \%$ da nota final;

- Para cada item do checklist, o aluno podia selecionar uma de quatro opções: "sim, totalmente", quando entendesse que o item estava completamente correto; "sim, largamente", quando entendesse que havia um pequeno erro ou melhoria a ser feita; "sim, parcialmente", quando entendesse que havia um erro maior, mas havia partes corretas ou "não", quando entendesse que o trabalho não atendeu em nada ao que foi solicitado. O objetivo de se estratificar a avaliação foi considerar uma escala de avaliação em vez dos itens tradicionais como "sim" e "não";

- A atribuição da nota da avaliação em par foi toda calculada pelo AVA. O AVA considera que o aluno recebe mais pontos quanto mais convergente for a avaliação em relação aos outros pares. Entretanto, os alunos foram orientados a realizar a avaliação de maneira fiel e correta. Caso o professor percebesse que as avaliações estavam todas excessivamente positivas (quando não deveriam), ele poderia não só atribuir uma nota específica ao trabalho avaliado como também atribuir nota zero aos alunos como avaliadores;

- Sugeriu-se ainda que o checklist fosse usado por cada aluno para aferir a qualidade de seu próprio trabalho antes da sua entrega;

- Foi facultativo ao professor alterar a nota total calculada pelo AVA como média das avaliações dos pares, sempre que ele entendesse procedente.

Ao final da disciplina, os alunos foram convidados a preencher um questionário de avaliação da mesma. Tal questionário contou com itens específicos para estudo da avaliação em pares na disciplina.

\section{Resultados e Discussões}

Esta seção apresenta a descrição do trabalho utilizada na condução das atividades em grupo que foram propostas aos alunos. A atividade aberta foi realizada dentro do contexto das disciplinas ofertadas no segundo semestre de 2015. Ao final da realização das disciplinas, dos 91 alunos matriculados, 76 realizaram a atividade. Todos eles foram convidados a responder a um questionário de avaliação do trabalho. Foram obtidas 30 respostas (39,5\% do total dos alunos que entregaram) e este resultado bem como sua análise são apresentados na Tabela 1 e discutidos a seguir.

Pode-se observar que ao menos $80 \%$ dos alunos respondentes classificaram os itens do checklist de avaliação em pares como ao menos razoavelmente completos e claros. Quanto à contribuição das avaliações em pares para o aprendizado e melhoria da 
qualidade dos trabalhos, $60 \%$ dos alunos a consideraram alta ou muito alta. A maioria dos respondentes $(83,3 \%)$ também entendeu que as avaliações realizadas pelos colegas podem ser classificadas em justas ou muito justas. A grande maioria dos alunos $(96,6)$ relatou que apresentou alto ou muito alto empenho para realizar a avaliação dos trabalhos dos colegas. Além destes resultados positivos, a maioria dos respondentes $(83,3 \%)$ entendeu que avaliar o trabalho dos colegas teve uma contribuição alta ou muito alta para seu próprio aprendizado. Por fim, a maioria dos respondentes $(73,3 \%)$ gostaria de utilizar a avaliação em pares em outras disciplinas do curso.

Tabela 1. Resultados do Questionário Aplicado

\begin{tabular}{|l|r|}
\hline \multicolumn{1}{|c|}{ Questão / Alternativas } & Respostas \\
\hline $\begin{array}{l}\text { Você considera que os itens do checklist para realização da avaliação em pares dos } \\
\text { trabalhos dos colegas foram }\end{array}$ & $30,0 \%$ \\
\hline Completos e claros & $50,0 \%$ \\
\hline Razoavelmente completos e claros & $20,0 \%$ \\
\hline Pouco completos e claros & $0,00 \%$ \\
\hline Nada completos e claros & \\
\hline $\begin{array}{l}\text { Você considera que a contribuição das avaliações realizadas pelos colegas em relação a } \\
\text { seu trabalho para a melhoria do mesmo e seu aprendizado foi }\end{array}$ \\
\hline Muito alta & $20,0 \%$ \\
\hline Alta & $40,0 \%$ \\
\hline Baixa & $30,0 \%$ \\
\hline Muito Baixa & $10,0 \%$ \\
\hline Você considera que a avaliação realizada pelos seus colegas foi & $3,3 \%$ \\
\hline Muito justa & $80,0 \%$ \\
\hline Justa & $16,7 \%$ \\
\hline Injusta & $0,0 \%$ \\
\hline Muito injusta & $43,3 \%$ \\
\hline Você considera que seu empenho em realizar a avaliação dos trabalhos dos colegas foi \\
\hline Muito alto & $53,3 \%$ \\
\hline Alto & $3,4 \%$ \\
\hline Baixo & $0,0 \%$ \\
\hline Muito Baixo & $10,0 \%$ \\
\hline $\begin{array}{l}\text { Você considera que a contribuição de realizar avaliações dos trabalhos dos colegas para } \\
\text { seu aprendizado foi }\end{array}$ \\
\hline Muito alta & $33,3 \%$ \\
\hline Alta & $50,0 \%$ \\
\hline Baixa & $73,3 \%$ \\
\hline Muito Baixa & $26,7 \%$ \\
\hline Você gostaria que a avaliação em pares fosse utilizada em outras disciplinas do curso? \\
\hline Sim & \\
\hline Não & \\
\hline & \\
\hline
\end{tabular}

Ao final do questionário, os alunos foram perguntados se teriam alguma sugestão de melhoria para o mecanismo de avaliação em pares usado no trabalho. 
Como comentários positivos, pode ser citado o aluno que afirmou "gosto da proposta da avaliação de pares, pois podemos ver os trabalhos dos outros colegas". Vários alunos questionaram o cálculo da nota, pois o sistema atribui a nota considerando o quão coerente a nota atribuída por cada aluno foi em relação às notas atribuídas pelos outros revisores do mesmo trabalho. Um aluno chegou a questionar este cálculo dizendo que "o cálculo de desvio que é feito em relação à nota que você dá a um colega e a avaliação dos outros alunos que o avaliaram é injusto". Outros alunos apresentaram sugestões de melhoria, por exemplo, "disponibilizar para o aluno uma solução completa da atividade do ponto de vista do professor" ou ainda "um campo para que o avaliador desse um feedback pelo motivo da escolha de cada opção". Por fim, houve comentários descrentes da eficácia da avaliação em pares. Um aluno chegou a afirmar que "infelizmente, a avaliação em pares não funciona em nosso sistema acadêmico, visto que os próprios alunos não se empenham em valorizar tal possibilidade de avaliação".

Em função dos comentários na última pergunta do questionário terem sugerido um conjunto de revisões e questionamentos sobre o método de avaliação em pares, decidiu-se buscar e avaliar mais profundamente alguns dados da avaliação da atividade. Apesar de a grande maioria dos alunos ter afirmado empenho na realização da avaliação em pares, buscaram-se indícios que pudessem afirmar o contrário. A Tabela 2 apresenta dados da participação dos alunos na avaliação em pares. Para cada disciplina, foi coletado o número de alunos que não avaliaram nenhum dos trabalhos designados a ele, o número de alunos que avaliaram todos os trabalhos designados a ele com nota máxima, e ainda o número de alunos que avaliaram o trabalho dos colegas sem ter realizado a entrega de seu próprio trabalho.

Tabela 2. Participação dos alunos na avaliação em pares

\begin{tabular}{|l|r|r|}
\hline & $\begin{array}{c}\text { Modelagem de } \\
\text { Aplicações Web }\end{array}$ & $\begin{array}{c}\text { Construção de } \\
\text { Aplicações Web }\end{array}$ \\
\hline $\begin{array}{l}\text { Número de alunos que não avaliaram } \\
\text { nenhum trabalho }\end{array}$ & $20(26,3 \%)$ & $16(21,1 \%)$ \\
\hline $\begin{array}{l}\text { Número de alunos que avaliaram todos os } \\
\text { trabalhos designados com nota máxima }\end{array}$ & $17(22,4 \%)$ & $30(39,5 \%)$ \\
\hline $\begin{array}{l}\text { Número de alunos que avaliaram os } \\
\text { trabalhos dos colegas sem ter realizado } \\
\text { entrega }\end{array}$ & $4(5,3 \%)$ & $2(2,6 \%)$ \\
\hline
\end{tabular}

O número de alunos que não avaliaram nenhum trabalho reflete o interesse e dedicação dos alunos à avaliação por pares. O número obtido nesta análise é bastante próximo ao número de alunos que não entregaram o trabalho, mostrando que possivelmente, trata-se de alunos desistentes do curso, mas ainda matriculados. $\mathrm{O}$ número de alunos que avaliaram todos os trabalhos designados com nota máxima pode ser um indicativo de baixo empenho na avaliação dos trabalhos, uma vez que os alunos podem apresentar desconforto, atitudes defensivas ou ainda comportamentos não totalmente sinceros por admirarem ou terem amizade com os colegas, conforme relataram Carlson, Berry e Voltmer (2005). Entende-se que, sobretudo, na disciplina de Construção de Aplicações Web, o fato de 30 alunos ou quase $40 \%$ dos 
participantes, avaliarem todos os trabalhos designados com nota máxima, pode ser considerado um forte indicativo de que as avaliações não foram de fato realizadas e estes alunos optaram ou até mesmo combinaram de atribuir nota máxima a todos. Vale destacar que estes números são tratados como indícios e futuras investigações mais profundas são necessárias. Por fim, o número de alunos que avaliaram os trabalhos dos colegas sem ter realizado entrega também pode ser visto como um indicativo de baixo empenho para a avaliação em pares, pois sequer houve a dedicação para entregar sua própria atividade. Poderia ser considerado indício de tentativa de ao menos se obter parte da nota da atividade. Entretanto, o número de alunos nesta situação foi baixo em ambas as disciplinas.

Outro dado interessante é o número de contestações de notas realizadas por alunos pelo fórum de discussão, ou seja, os alunos entenderam que a avaliação dada pelo revisor de seu trabalho não foi correta ou justa. Houve apenas 4 contestações em ambas as disciplinas. Neste caso, o professor verificou o trabalho do aluno e ajustou a nota, quando necessário.

\subsection{Lições Aprendidas}

Observando as discussões realizadas nos fóruns das disciplinas e a avaliação dos dados obtidos com o questionário apresentado anteriormente, foi possível identificar algumas lições aprendidas assim como ações a serem implantadas na próxima oferta das disciplinas:

- Foi observada a importância de se ter uma rubrica completa, de fácil entendimento e alinhada aos conteúdos da disciplina para avaliação dos trabalhos. Uma rubrica com estas características reduz significativamente as perguntas e dúvidas dos alunos;

- São necessários mecanismos mais eficientes de controle e verificação de quais alunos de fato realizaram a avaliação por pares;

- O uso do AVA, no caso o Moodle, contribui para a automatização e redução dos esforços no gerenciamento da atividade;

- Os checklists de verificação devem estar disponíveis desde o início da realização da atividade para que os alunos conheçam os critérios aos quais a avaliação de seu trabalho estará submetida;

\section{Conclusões e Trabalhos Futuros}

Este trabalho apresentou o estudo de um caso de aplicação da técnica de avaliação em pares como mecanismo de avaliação em um curso de especialização à distância. Entendese que a adoção deste mecanismo de avaliação se insere em um contexto de aprendizagem colaborativa, ou seja, no qual os alunos podem colaborar e cooperar entre si, com o apoio do professor, para promover seu próprio aprendizado. Segundo Costa e Franco (2006), a aprendizagem colaborativa é um caminho natural para as iniciativas em EaD. Entende-se também que a avaliação por pares é uma prática de avaliação formativa, pois permite ao aluno desenvolver a habilidade crítica de revisão do trabalho bem como ampliar seu aprendizado com uma solução diferente para o mesmo problema 
que resolveu e ainda refletir sobre as suas estratégias. Segundo Perrenoud (1999) a avaliação formativa aposta na capacidade do sujeito para gerir ele próprio seus projetos, seus progressos, suas estratégias diante das tarefas e dos obstáculos.

O estudo da avaliação em pares foi realizado por meio do relato das experiências dos alunos por meio de um questionário e também pela análise de dados do próprio curso estudado. No questionário, os alunos na sua maioria consideraram a avaliação em pares realizada pelos colegas como justa ou muito justa e ainda relataram que tanto receber os comentários dos colegas quanto o fato de poder revisar os trabalhos dos mesmos contribui com seu aprendizado. Entretanto, existem indícios que uma parte considerável dos alunos não se dedicou completamente à avaliação por pares, pois, por exemplo, eles atribuíram a mesma nota a todos os trabalhos que avaliaram.

Este trabalho contribui para o campo de pesquisa em EaD uma vez que foram identificados poucos trabalhos na literatura sobre a adoção de avaliação em pares e ainda, a grande maioria deles, a utiliza para a avaliação de textos somente. Este trabalho se diferenciou dos anteriores por aplicar a avaliação em pares em um contexto de curso de especialização à distância na área de Tecnologia da Informação, pela avaliação da percepção dos alunos quanto a este instrumento e pela discussão de dificuldades enfrentadas quanto à garantia do empenho dos alunos nesta atividade. Acredita-se que o uso destas avaliações deva ser incentivado e seu processo evoluído de forma a contribuir para o aprendizado dos alunos e também ser uma ferramenta de efetiva avaliação a ser utilizada pelos professores.

Como trabalhos futuros, pretende-se incorporar as sugestões dadas pelos próprios alunos e buscar um mecanismo mais eficiente de controle e acompanhamento das revisões para reduzir a possibilidade de o aluno a realizar sem o empenho necessário. Pretende-se ainda gravar vídeo-aulas explicando o processo e apresentando um exemplo do uso de avaliação em pares.

\section{Referências}

BALULA, A. Avaliação das Aprendizagens em contexto de eLearning, Nov@ Formação - eLearning e Avaliação - Métodos e Indicadores. Lisboa: Inofor, 6, pp.710, dez, 2005.

BALULA, A. Avaliação como aprendizagem em ambiente on-line. Linhas Críticas, V.19, n.40, pp. 505-522, 2013.

CARLSON, P.; BERRY, F. C.; VOLTMER, D. Incorporating Student Peer-Review into an Introduction to Engineering Design Course. In: Proceedings of the 35th ASEE/IEEE Frontiers in Education Conference, 2005, Indianopolis, EUA. Anais. Indianopolis: IEEE.

CHETWYND, F.; GARDNER, C.; JEFFERIS, H. Literature Review for the eSTEeM project "The use of peer assessment/review in distance teaching via the Moodle VLE”. Relatório final, 2013. Disponível em <http://www.open.ac.uk/about/teachingand-learning/esteem/projects/themes/innovative-assessment/the-use-peerassessmentreview-distance-teaching-the-moodle-vle>. Acesso em 16-04-2016. 
COSTA, L. A. C.; FRANCO, S. R. K. Aprendizagem Colaborativa na Educação A Distância: Aspectos Teóricos, Estratégias Pedagógicas e Experiências Realizadas. Revista Novas Tecnologias na Educação (RENOTE), v.4, n.2, 2006.

GARCIA-ALMIRALL, P.; REDONDO, E.; VALLS, F.; FONSECA, D. Peer-review in architecture education: Application in Geographic Information Systems learning. In: Proceedings of the 10th Iberian Conference on Information Systems and Technologies (CISTI), pp.1-7, 2015, Aveiro, Portugal. Anais. Aveiro: AISTI.

GIELEN, S., DOCHY, F., ONGHENA, P., STRUYVEN, K. and SMEETS, S. Goals of peer assessment and their associated quality concepts. Studies in Higher Education, v. 36, n. 6, 2011.

MUHSEN, Z. F.; MAAITA, A.; ODAH, A.; NSOUR, A. Moodle and e-learning Tools. International Journal of Modern Education and Computer Science, v.6, pp.1-8, 2013.

NASCIMENTO, S. B.; FERREIRA, S. M. B.; CORDEIRO, B. M. P. Auto e HeteroAvaliação Docente: Uma Ferramenta da Gestão de Desempenho Aplicada ao Ensino Superior na Modalidade a Distância. $\mathbf{1 7}^{\mathbf{0}}$ Congresso Nacional de Educação à Distância, 2011, Manaus, Amazonas. Anais. Manaus: ABED

PAPADOPOULOS, P. M.; PANTELIS M.; LAGKAS, T. D.; DEMETRIADIS, S. N. How to Implement a Technology Supported Free-Selection Peer Review Protocol Design Implications from Two Studies on Computer Network Education. In: Proceedings of the 12th IEEE International Conference on Advanced Learning Technologies, 2012, Roma, Itália. Anais. Roma: IEEE.

PERRENOUD, P. Avaliação: da excelência à regulação das aprendizagens - entre duas lógicas. Porto Alegre: Artes Médicas Sul, 1999.

SOFTEX. Guia de Implementação do Nível D do MPS.Br, 2016. Disponível em < http://www.softex.br/wpcontent/uploads/2013/07/MPS.BR_Guia_de_Implementacao_Parte_4_2016-1.pdf>. Acesso em 22-04-2016.

SONG, W.; SIRILUCK, U. How EFL university students use electronic peer response into revisions. Suranaree Journal of Science \& Technology, Vol. 16 N.3, 263-275, 2009.

YIN, R. K. (1984). Case study research: design and methods. London: Sage.

YOSHIZAWA, S.; TERANO, T.; YOSHIKAWA, A. Assessing the Impact of Student Peer Review in Writing Instruction by Using the Normalized Compression Distance. IEEE Transactions on Professional Communication, vol. 55, n. 1, 2012.

ZENHA-RELA, M.; CARVALHO, R. Work in Progress: Self Evaluation Through Monitored Peer Review Using the Moodle Platform. In: Proceedings of the 36th Frontiers in Education, pp. 26-27, 2006, San Diego, California, EUA. Anais. San Diego: IEEE. 